\title{
Explainer: An interactive Agent for Explaining the Diagnosis of Cardiac Arrhythmia Generated by IK-DCBRC
}

\author{
Type of article: Original \\ Abdeldjalil KHELASSI1, Vania V Estrela2, Jude Hemanth3 \\ 1 Abou Bakr Belkaid University of Tlemcen, Algeria \\ 2 Fluminense Federal University (UFF), Brazil \\ 3 Karunya University, Coimbatore, India
}

\begin{abstract}
Interactions between medical applications and users involve a high level of trust, since many complex, automated applications are integrated and involve critical domains in which public health is paramount. Although uncertainty decreases the accuracy and trust of such medical applications under these circumstances, explanation-aware computing becomes crucial in improving the efficiency of these applications. This paper describes an intelligent agent that interacts with users to provide meaningful explanations of previous diagnoses supported by IK-DCBRC. The agent ensures intelligent interactions with users via a rule-based system that generates appropriate explanations according to the selected level of abstraction and the detected cardiac arrhythmia. The paper also describes a particular medical application, that is, cardiac arrhythmia with automatic diagnoses supported by the case-based reasoning classifier, IK-DCBRC.

Keywords: Case-based reasoning, Explanation-aware computing, Cardiac arrhythmias, Intelligent agent.

Corresponding author: Abdeldjalil KHELASSI Abou Bakr Belkaid University of Tlemcen, Algeria khelassi.a@ gmail.com Received: 15 May, 2019, Accepted: 27 June, 2019, English editing: 22 May, 2019, Published: 13 July, 2019.

Screened by iThenticate..@2017-2019 KNOWLEDGE KINGDOM PUBLISHING.
\end{abstract}

\section{Introduction}

The primary cause of deaths worldwide is through cardiovascular diseases, which accounted for 17.65 million deaths in 2018 [1]. The detection and classification of heart arrhythmia using electrocardiogram (ECG) signals comprise an active area of research [2]. Several techniques have been successfully implemented for the detection of cardiac arrhythmia, including the case-based reasoning (CBR) [3] support vector machine (SVM) [4] and artificial neuronal network (ANN)[5]. Although there have been several achievements in this area, each technique possesses some weaknesses that may increase the risk caused by the uncertainty of the domain and the applied measurement techniques. This risk decreases the trust and credibility of the detection system.

The uncertainty is a common problem between many research domains. It is defined in several contexts as predictions of future events, physical measurements, etc. The Fuzzy Sets Theory is introduced for resolving the problem of uncertainty by extending the classical set theory since, for a certain universe, a membership function may act as an indicator function, mapping all elements to either 1 or 0 , as in the classical notion [6].

Case-Based Reasoning is a successful method for problem solving and classification. It consists of solving problems based on remembering anterior analog cases and by reusing or matching information and knowledge of those cases [7]. Several CBR systems and frameworks were implemented and applied for resolving several problems as, myCBR, and JCOLIBRI [8]. In this paper a self-developed 
CBR system IK-DCBRC (Intensive Knowledge-Distributed Case-Based Reasoning Classifier) is used, which is successfully applied in medical decision support and diagnosis, it ensures promising results of classification and clustering, it also includes an original fuzzy similarity measure function which considerably improves the precision of the system (see [3]).

CBR confronts several problems since they are open-ended, and often dynamic, including uncertainty, model incompleteness, and deficient problem descriptions [9]. This makes the explanation an indispensable option or component of CBR systems for increasing the trust between these complex systems and their users. Several explanation techniques in different case-based reasoning systems have been reviewed in [9], in which an extensive survey regarding explanation theory, requirements, and models is presented.

This paper describes an original interaction agent; the goal planned by this agent is to visualize an explanation-aware medical diagnosis supported by IK-DCBRC. In this agent, we introduce the notion of "multi-level of abstraction", a rule-based system, and traces of prior diagnoses for intelligent interaction. The users select the level of abstraction. The agent addresses all required explanation types, but uses just the appropriate one; the explanation is visualized according to the selected level of abstraction.

The remainder of this paper describes the cardiac arrhythmia and the physiological ECG signal, the explanation in intelligent systems and concisely the IK-DCBRC system. Finally, it presents the implemented interaction agent with the used approach and experimental validation of explanations.

\section{Cardiac arrhythmia diagnosis}

Cardiac arrhythmia accounts for a group of disorders in which the electrical activity of the heart is irregular. There are more than forty cardiac arrhythmias detected by ECG (see Fig 1). Significant reviews of ECG, which are published in [10] also, [11] and [12], could be useful for additional information. The Automatic measurement of ECG parameters is an essential area for applying some computational and signal analysis methods as in [12, 13 and 14] for wave detection and measurements. The automatic recognition and diagnosis of cardiac arrhythmia is an active research domain, where precision and transparency are crucial. In the literature, we find a diversity of approaches and intelligent techniques successfully applied as the artificial neuronal networks ANN [5], support vector machines SVM [4], fuzzy approaches [15], case-based reasoning [3], and hybrid models [16].

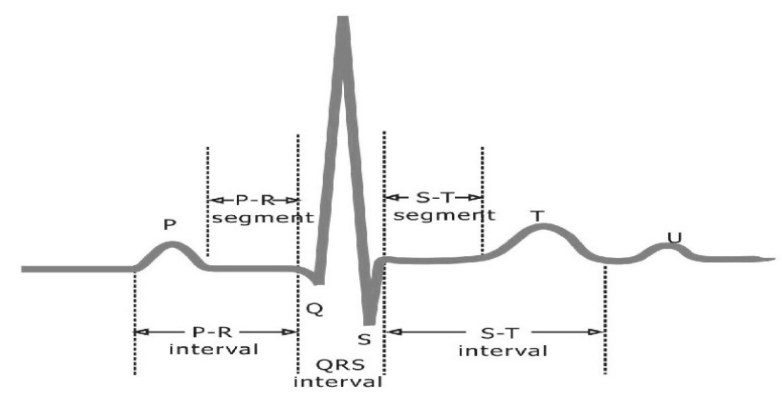

Fig. 1.The ECG. 


\section{IK-DCBRC: The medical decision support system}

Case-based reasoning is a successful paradigm implemented for the resolution of several problems as information retrieval, recommendation systems, clustering, and classification. It consists of resolving new problems by remembering the prior resolution of similar cases [7]. Several trends and categorization of CBR are well described in [17] and [18].

The distributed variant of CBR is commonly applied for improving the performance of several systems, for example [19, 20, 21, and 22]. Although, the implementation was realized in four distinguished forms [18] through two criteria: knowledge organization, and knowledge inference process.

We have developed a distributed case-based reasoning system IK-DCBRC, which is classified as multi-case bases and multi-processing agents. We applied it for medical decision support [23] and data mining [24]. IK-DCBRC became an interesting tool for several cognitive experiments and applications. It can be used in several cognitive scenarios in different domains. As shown in Fig2 and Fig 3, IK-DCBRC implements a distributed reasoning variant with a set of cognitive and interactive agents. In addition, several techniques of knowledge extraction from data can be integrated through the implemented algorithms and its extensibility. It was successfully applied in two valuable medical domains: cardiac arrhythmia [3, 23, 26, 29,30 and 31] and breast cancer diagnosis [24, 25, and 32].

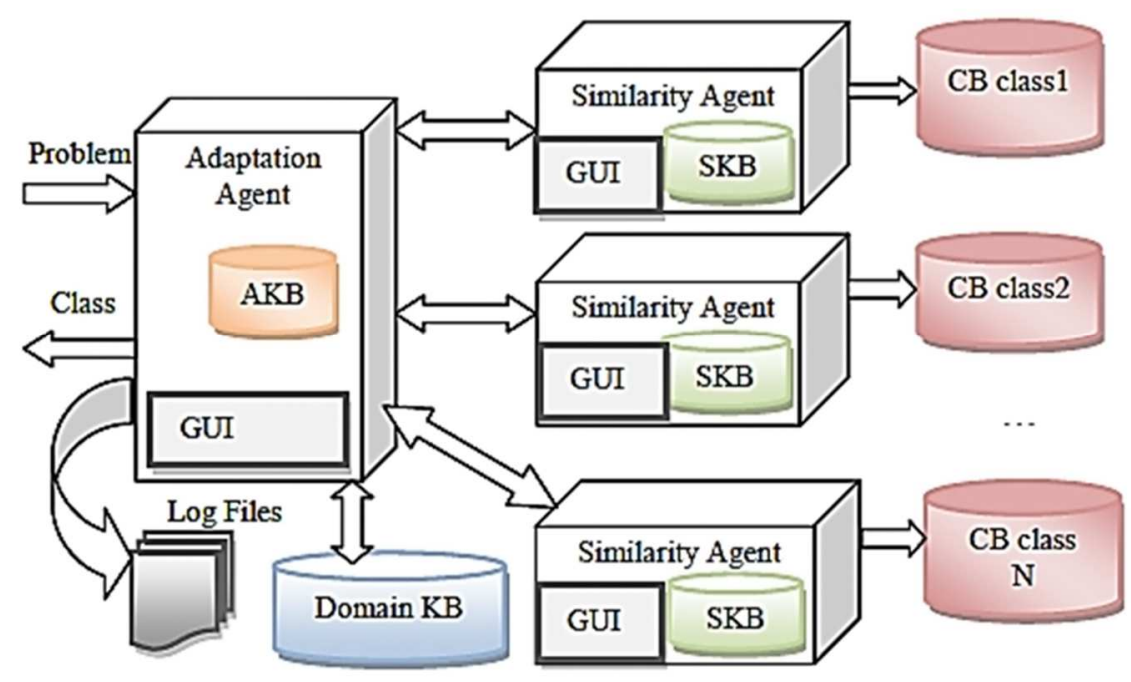

Fig. 2. IK-DCBRC: Distributed decision support system

Fig 2 explains the most critical components of IK-DCBRC. The distributed architecture optimizes considerably the performance of the decision support system as well as the transparency, which also affects the maintainability. Two kinds of cognitive agents are defined:

1. The similarity agent computes the rate of membership of the query in the associated case base of cardiac arrhythmia (the disease). This rate represents the aggregation of the similarity measures between the query and cases of 
the associated class case base, see formula (1). This cognitive agent extracts similarity knowledge (SKB) from the prior resolved cases, also by an interactive acquisition from users, (the users can select the similarity measures function and the weights definition methods). According to the proposed architecture $\mathrm{N}$ agents can be defined, but each one should be specialized for exactly one disease. Under these circumstances, the detection of conflicts can be achieved by the explanation agent.

2. The adaptation agent edits the query by interacting with the users, following that, it communicates with the similarity agents by sending a message containing the query associated with an ontology, which describes the features of this query. The adaptation is realized by inferring from adaptation knowledge base $\mathrm{AKB}$, represented as a set of rules, which defines the protocol of decision by weighting the responses of each cognitive agent. The reasoning is also enriched by a domain knowledge base, which contains the rules extracted from the experts' knowledge.

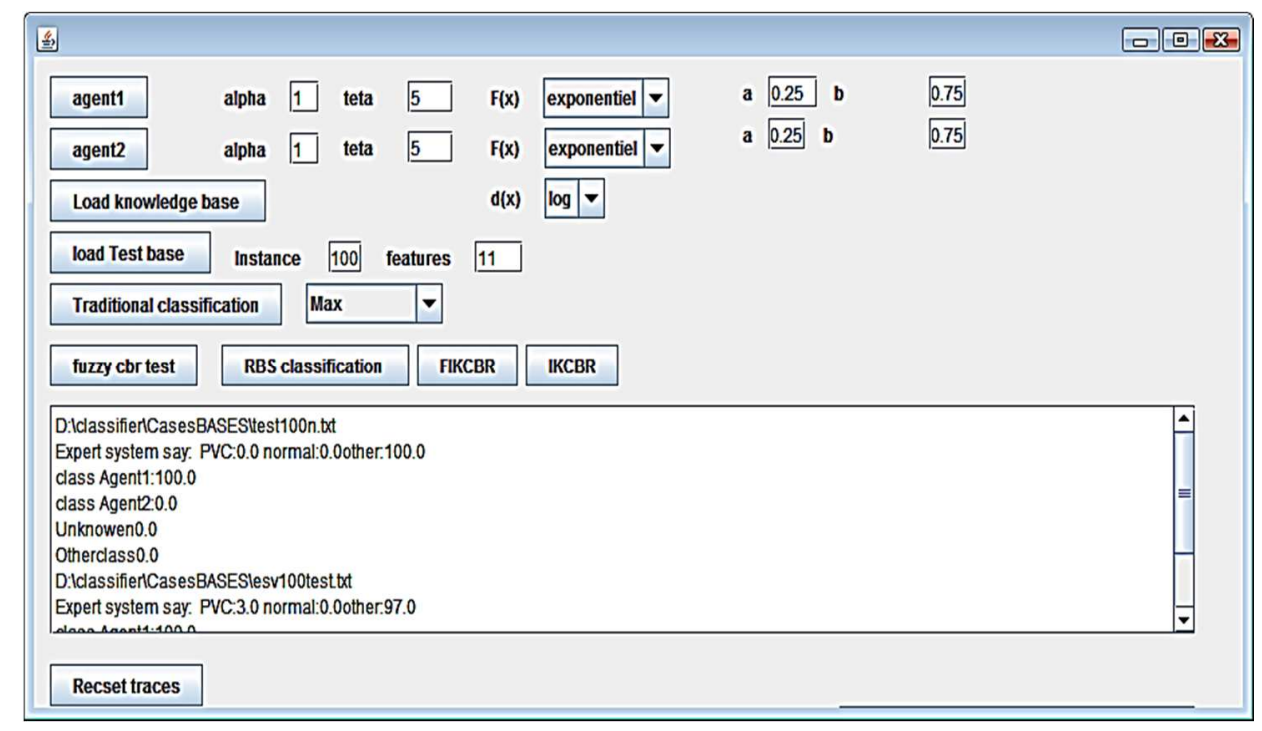

Figure 3.a Adaptation Agent GUI 


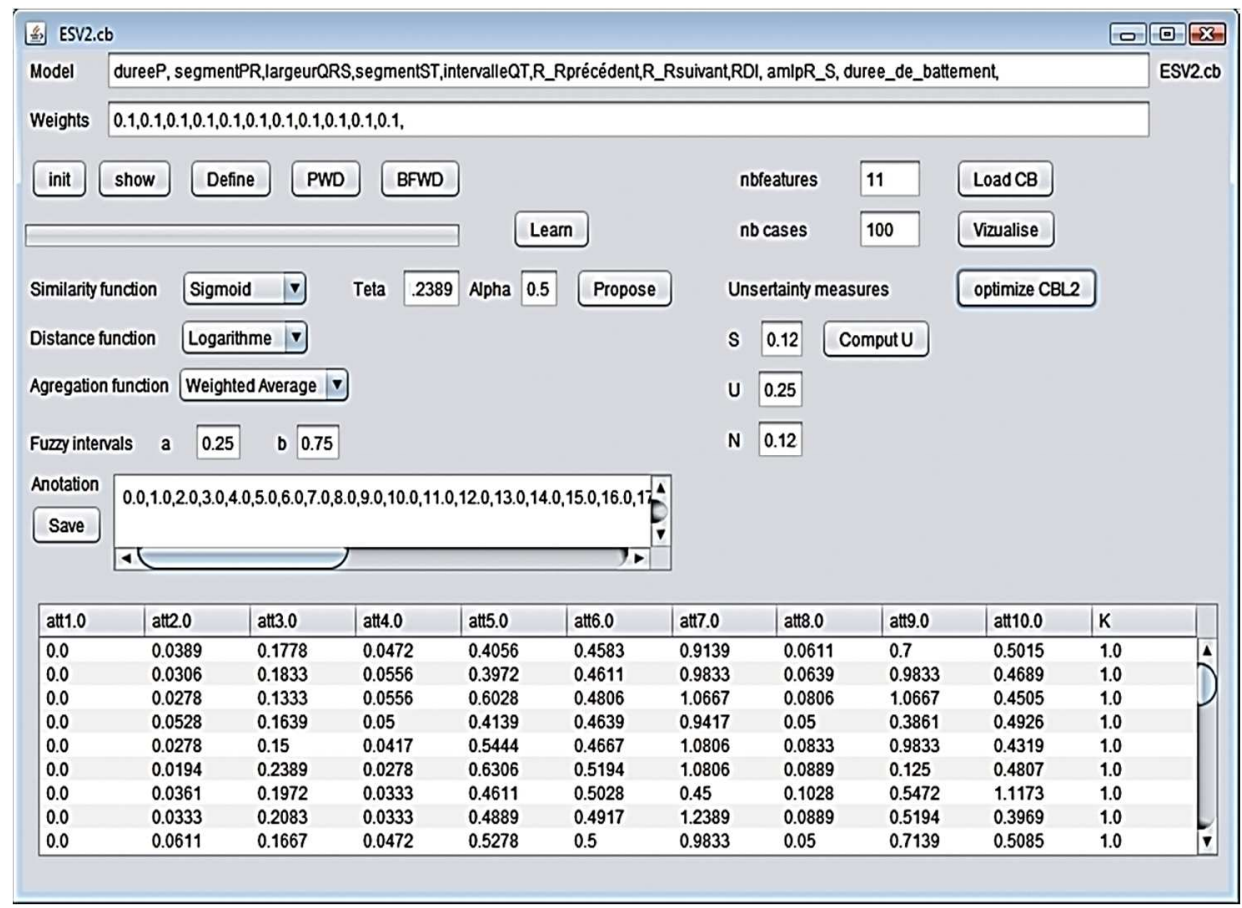

Figure 3.b Similarity Agent GUI

Fig. 3. IK-DCBRC Graphic User Interfaces: Adaptation agent and Similarity agent.

Log files generated by the developed system contain all needed information for explanations (see Table 2). They were extracted from the agents' messages as the computed similarity measures of each similarity agent, also considering the uncertainty of responses of the used adaptation rules and similarity parameters. These files can be visualized with text editor software, but the contained information is extensive, and many queries need to be treated by the reasoning system. For these reasons, a definition of a separated component for explanation is suitable.

\section{Similarity measures}

In the similarity measures' research domain, two approaches to compute the similarity are defined, namely: 1) the traditional one and 2) the local-global similarity measures one as cited in [23]. The first one is based on common mathematical principles and distance measures including Hamming Distance, Simple Matching Coefficient (SMC), weighted SMC, non-linear SMC, Tversky Contrast Model, City Block Metric, Euclidean Distance, and Maximum Norm...etc. The second one, which can be used in IK-DCBRC, is the local-global similarity measures. It is based on decomposing the similarity in local part computed between attributes values and a global, which aggregates all these locals. The role of the similarity measures is to simplify the modeling, and it allows defining of wellstructured measures even for very complex case representations consisting of numerous attributes with different value types. 
Definition1. (The weight vector) Let $\mathrm{D}=\left(\mathrm{A}_{1}, \mathrm{~A}_{2}, . ., \mathrm{A}_{\mathrm{n}}\right)$ be a case characterization model. The vector $\vec{W}=\left(\mathrm{w}_{1}, \mathrm{w}_{2}, . ., \mathrm{w}_{\mathrm{n}}\right)$ with wi $\in[0,1]$ and $\sum_{t=1}^{n} w_{i=1}$, is called weight vector for $D$, where each element $w_{i}$ is called attribute weight for $A_{i}$.

Definition2. The local similarity function for an attribute $A$ is a function $\operatorname{Sim}_{\mathrm{A}}: \mathrm{A}_{\text {range }} \times \mathrm{A}_{\text {range }} \rightarrow[0,1]$.

With $A_{\text {range }}$ is the value range of attribute $A$.

There are many developed similarity functions for the local measures: linear, threshold, exponential, sigmoid, Co-sinus and other similarity functions, which permit the computation of local similarity functions between two attributes within the same domain.

Some similarity functions use the Euclidian distance others use the logarithmic distance for numerical attributes and the similarity table or ontological distance for symbols [23].

Definition3. (The global similarity function)

Let $\mathrm{D}=\left(\mathrm{A}_{1}, \mathrm{~A}_{2}, \ldots, \mathrm{A}_{\mathrm{n}}\right)$ be a case characterization model, $\mathrm{w}$ be a weight vector, and $\operatorname{sim}_{i}$ be a local similarity measure for the attribute $A_{i}$. A global similarity measure for $\mathrm{D}$ is a function

$\operatorname{Sim}: \mathrm{D} \times \mathrm{D} \rightarrow[0,1]$

$\operatorname{Sim}(\mathrm{Q}, \mathrm{C})=\pi\left(\operatorname{sim}_{1}\left(\mathrm{q}_{1}, \mathrm{c}_{1}\right), \ldots, \operatorname{sim}_{\mathrm{n}}\left(\mathrm{q}_{\mathrm{n}}, \mathrm{c}_{\mathrm{n}}\right), \mathrm{w}\right)(4)$

Where $\pi:[0,1]^{\mathrm{n}} \rightarrow[0,1]$ is called aggregation function that must fulfil the following properties: $\forall \vec{w}: \pi(0, \ldots, 0, \vec{w})=0 \pi$ is increasing monotonously in the arguments representing local similarity values. There are many defined aggregation functions $\pi$. Examples of commonly used ones are:

$\pi\left(\operatorname{sim}_{1}, \ldots, \operatorname{sim}_{n}, \vec{w}\right)=\sum_{i=1}^{n} w \cdot \operatorname{sim}_{i}$ Weighted Average Aggregation

$\pi\left(\operatorname{sim}_{1}, \ldots, \operatorname{sim}_{n}, \vec{w}\right)=\left(\sum_{i=1}^{n} w \cdot \operatorname{sim}_{i}^{p}\right)^{1 / p}$ Minkowski Aggregation

$\pi\left(\operatorname{sim}_{1}, \ldots, \operatorname{sim}_{n}, \vec{w}\right)=\max _{i=1}^{\pi l} w \cdot \operatorname{sim}_{i}$ Maximum Aggregation

$\pi\left(\operatorname{sim}_{1}, \ldots, \operatorname{sim}_{n}, \vec{w}\right)=\min _{i=1}^{n} w \cdot \operatorname{sim}_{i}$ Minimum Aggregation

For ensuring meaningful and accurate reasoning, we have introduced a fuzzy similarity model, which is published in [3]. The membership functions are defined as follows:

$\mu_{s}(x)=\left\{\begin{array}{c}0 \text { if } x \leq a \\ \frac{x-a}{1-a} \text { if } x>a\end{array}\right.$

$\mu_{n}(x)=\left\{\begin{array}{l}0 \text { if } x \geq b \\ \frac{b-x}{b} \text { if } x<b\end{array}\right.$

$\mu_{u}(x)=\left\{\begin{array}{c}0 \text { if } x \leq b \text { ou } x>a \\ \frac{x-b}{0.5-b} \text { if } x>b \text { and } x \leq 0.5 \\ \frac{a-x}{a-0.5} \text { if } x<a \text { and } x>0.5\end{array}\right.$

The variable $x$ represents the result of the similarity aggregation function. The supports of the fuzzy sets ( $a$, b values) are defined by using the GUI of the similarity agent or by using a machine learning algorithm. 


\section{Example of uses}

As presented in the sequence diagram in Fig4, the adaptation agent edits the following cardiac beat characteristics and its ontology:

Giving the following characteristics of a cardiac beat example (diagnosed as Premature ventricular contraction PVC):

$(0.0000,0.1489,0.1778,0.0472,0.4056,0.4583,0.9139,0.0611,0.7000,0.5015, ?)$

And the associated pattern:

(dureeP,segmentPR,largeurQRS,segmentST,intervalle $Q T, R \_R p r e ́ c e ́ d e n t, R \_R s u i v a$ $n t, R D I, a m l p R \_S, d u r e e \_d e \_b a t t e m e n t$, class).

More details about this pattern can be found in [3 and 23].

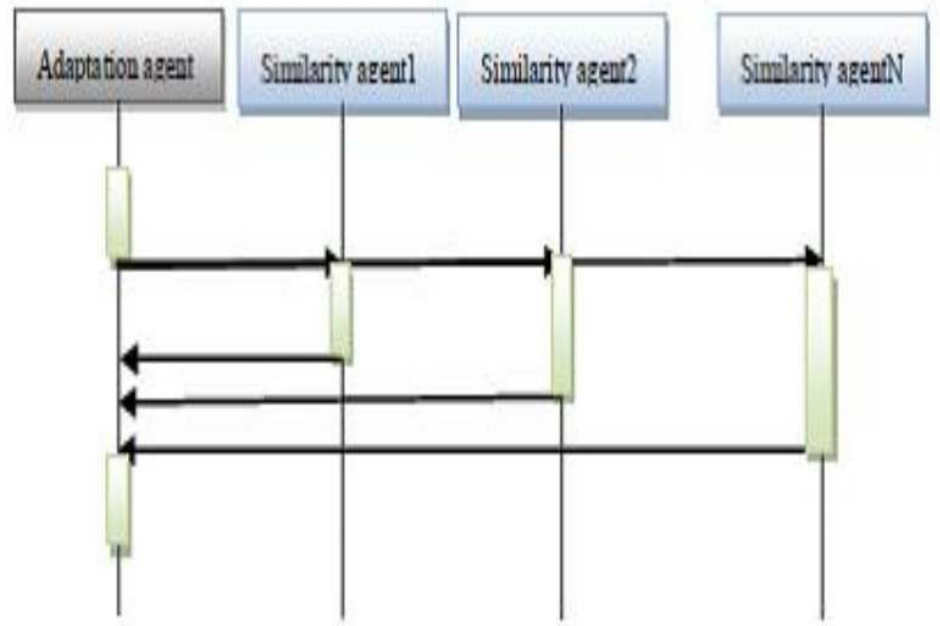

Fig.4. The sequence diagram of IK-DCBRC

The similarity agents receive the request and compute the membership of the query in the class by using their learned knowledge and the mean values of the similarity measures, see (5), (6), and (7). More details are published in [3, 24]. The adaptation agent, in this example, received the following messages:

\section{Agent 1 response:}

Similar: 22\%, not-similar: $68 \%$, unknown: $10 \%$.

Agent 2 response:

Similar: $28 \%$, not-similar:00\%, unknown: $72 \%$.

Rule-based system response:

Unknown

The last action is the inference from the adaptation knowledge base for the seven received parameters.

The response of the adaptation agent (the class of this beat) is: $P V C$. 


\section{Empirical experiments}

The first results published in [23 and 27], by applying just the local-global similarity measures, did not ensure the trust of physicians. This was due to the low precision of the system: from $25 \%$ to $49 \%$ as the rate of correct classification with several aggregation functions. Nevertheless, these poor results encouraged us to apply the Intensive-Knowledge Case-Based Reasoning approach [27, 28] and the Fuzzy Sets Theory $[25,26]$ for augmenting the precision of the developed system.

IK-DCBRC is a personalized classification system in which several similarity measures can be used for the classification. As mentioned in [3], we have realized several experiments for the evaluation and validation of the developed system and the introduced fuzzy similarity model. The most relevant results are described in Table 1 . These results represent three experiments achieved in the classification of 400 cardiac beats.

Table.1. Results of experiments

\begin{tabular}{|l|c|c|}
\hline & Correct classification & Error \\
\hline Experiment 1 & 74.5 & 25.5 \\
\hline Experiment 2 & 77.25 & 22.75 \\
\hline Experiment 3 & 100 & 0 \\
\hline
\end{tabular}

Experiment 1 demonstrated that our rule-based system had significant uncertainty problems because the rate of correct classification was only $74 \%$. This is probably due to using rules only partially, or the measures of ECG parameters were approximate

Experiment2 improved the impact of Case-Based Reasoning against the rule-based system. However, uncertainty is still essential.

The best result, which represents entirely recognized arrhythmias, was the third experiment. This experiment improves the usefulness of applying fuzzy similarity measures and domain rules. This is due to the most substantial impact of the Fuzzy models resolving the uncertainty problems.

The accuracy of this system was also measured via a benchmark realized by two international medical data sets: MIT-BIH for the cardiac arrhythmia in [30] and a breast cancer dataset collected and published by Dr Wollberg in [24]. Other results and deep comparisons with the related works exist in [23-32].

\section{Explanations in intelligent systems}

The concept of explanation is a common area of interest in several related domains such as didactics, physics, cognitive systems, philosophy, and other domains. Intelligent systems can be viewed as an applied science of cognitive systems. Usually, these complex systems confront a wide variety of critical applications where the uncertainty represents a substantial risk. Under these circumstances, the explanation of intelligent systems behaviors becomes a necessity for a trusted interaction between the users and these complex systems.

The terminology of explanation in Artificial Intelligence (AI) takes two forms: 1) a process of reasoning as a necessary cognitive component, and 2) an additional component, which helps users to interpret and understand the cognitive system [33]. 
In the literature of explanation, an important systematic review [9] is published, in which a historical overview of the concept uses philosophy and AI. Two taxonomies of explanation are defined, the first one according to the users' goals (Transparency, Justification, Relevancy, Conceptualization, Learning) [34]. The second taxonomy [9] is defined according to the existing implementation of explanation in the artificial reasoning systems (see Table2).

Table2 Taxonomy of explanations from [9].

\begin{tabular}{|l|l|}
\hline Type & Example \\
\hline Reasoning Trace & MYCIN [35]. \\
\hline Justification & XPLAIN [36]. \\
\hline Strategic & NEOMYCIN [35]. \\
\hline Terminological & $\begin{array}{l}\text { WSwartout and SSmoliar } \\
\text { system [37]. }\end{array}$ \\
\hline
\end{tabular}

\section{Explainer}

\section{Conceptual model}

We have developed a cognitive agent for a multi-level explanation of the decision supported by IK-DCBRC. As shown in Fig 5, the explanation agent visualizes the appropriate explanation for the level of abstraction selected by the users. The agent ensures an intelligent interaction by inferring from the explanation knowledge base. This inference is for an appropriate explanation of the diagnosis supported and logged by IK-DCBRC. Three levels of abstraction are defined as an appropriate visualization of all kinds of explanation. Some related works in the use of explanation knowledge bases are described in [38, 39]. 


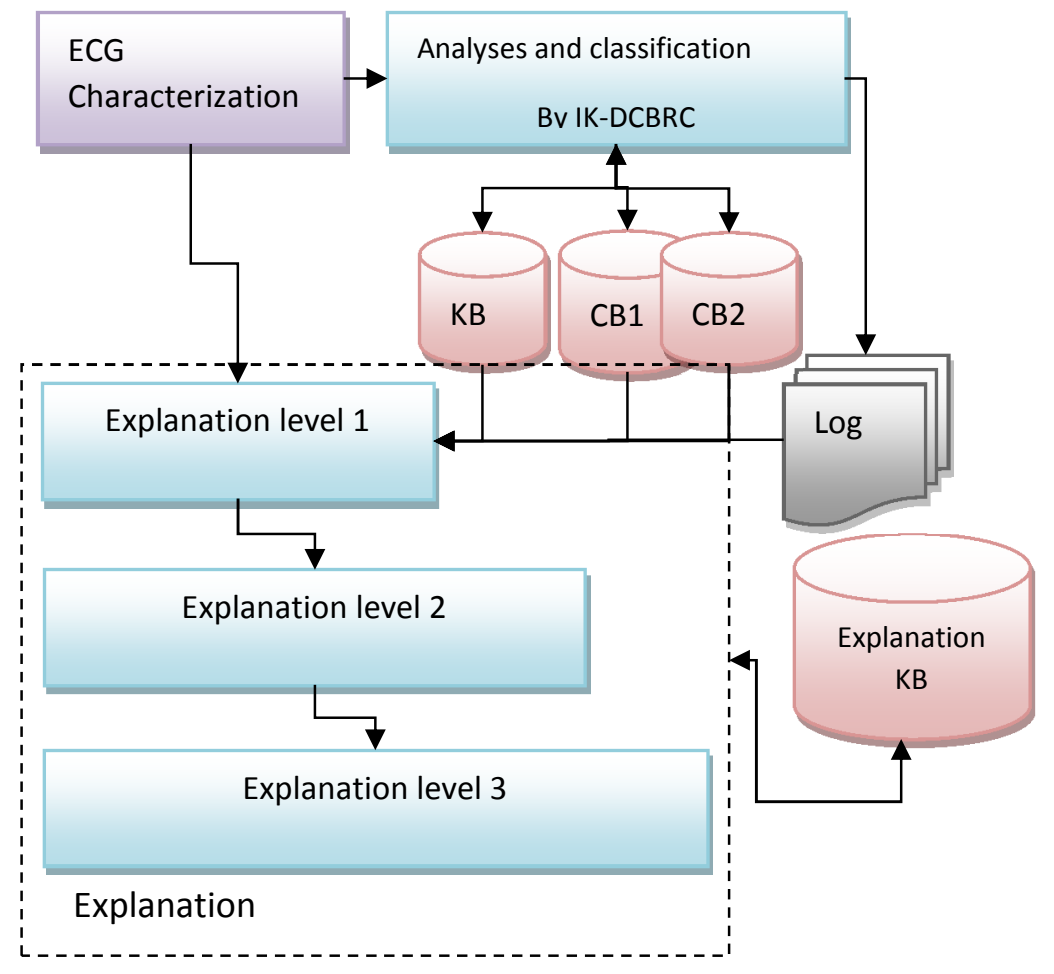

Fig.5. The explanation agent and its environment.

\section{The Graphical User Interface}

As presented in Fig 6, the explanation agent interface facilitates the definition of the level of abstraction, the selection of input files (the log file, the case bases, and the explanation knowledge base) and the visualization of explanations. There are three levels of abstractions: one for novice users, one for medical experts, and one for developers. The explanation agent should reuse some cases for ensuring the needed kind of explanation is adapted to that of the application users. The vital impact of a Fuzzy similarity model appears to be in the simplified method of presentation where the users understand the linguistic variables more than similarity values. Some web links such as wiki documents are used for explanations to novice users. The explanation agent also visualizes the ECG and an annotated image of the arrhythmia (for example, the PVC in Fig 5). 


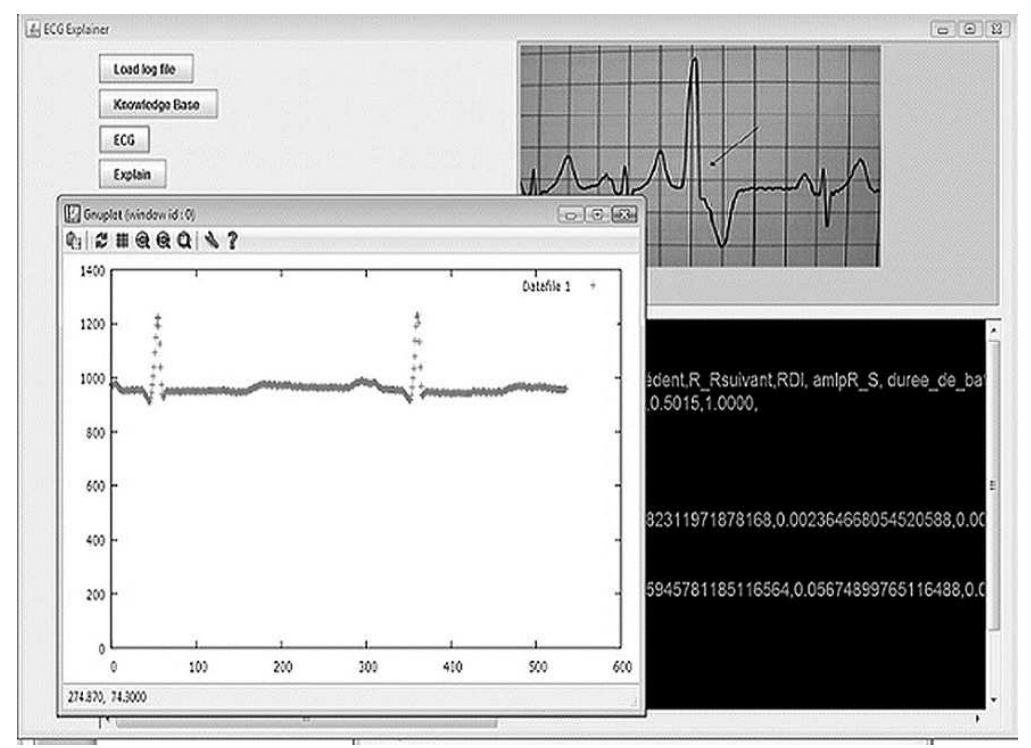

Fig. 6. The explanations agent user interface.

\section{The log file}

The log file constructed by the intelligent system contains the different useful information and reasoning traces about the decision inferred by the cognitive agents. Log file contents are included in Table 3.

Table3. The information logged by the DSS IK-DCBRC

\begin{tabular}{|c|c|c|}
\hline \multicolumn{2}{|l|}{ Features } & Description \\
\hline \multicolumn{2}{|l|}{ Query id } & The identification of the query \\
\hline \multicolumn{2}{|l|}{ Pattern } & List of the features of the query \\
\hline \multicolumn{2}{|l|}{ Query } & List of the values of the features of the query \\
\hline \multicolumn{2}{|l|}{ RBSrsp } & The response inferred from the expert system \\
\hline \multicolumn{2}{|l|}{ RBST } & $\begin{array}{l}\text { The trace of reasoning of the expert system (the } \\
\text { used rules). }\end{array}$ \\
\hline \multirow[t]{3}{*}{ SimAgent } & Sim & $\begin{array}{l}\text { Similarity function, distance function and their } \\
\text { parameters. }\end{array}$ \\
\hline & $\mathrm{Wi}$ & List of the features importance degree \\
\hline & Rsp & $\begin{array}{l}\text { Rate of membership in the fuzzy sets: S (similar), } \\
\text { U (unknown), N (not similar), or the global } \\
\text { similarity(if the agent used the traditional } \\
\text { similarity) }\end{array}$ \\
\hline \multirow[t]{2}{*}{ AdaptAgent } & Adrsp & The response of the adaptation \\
\hline & Adpttr & $\begin{array}{l}\text { The trace of reasoning in the adaptation(the used } \\
\text { rule for adaptation) }\end{array}$ \\
\hline \multicolumn{2}{|l|}{ Disease } & The detected disease \\
\hline
\end{tabular}

\section{Level of abstractions}

The cognitive agent for explanation defines the following three levels of abstraction selected by the users (Fig 7, 8, and 9): 


\section{- Level 1}

In this level, the agent visualizes an ordinary explanation for novice users. The agent analyses the log file and the explanation knowledge base. After that, the agent presents the disease, the terminological explanation and the indexed links of useful wiki documents and the annotated image of the cardiac beat, for consultation.

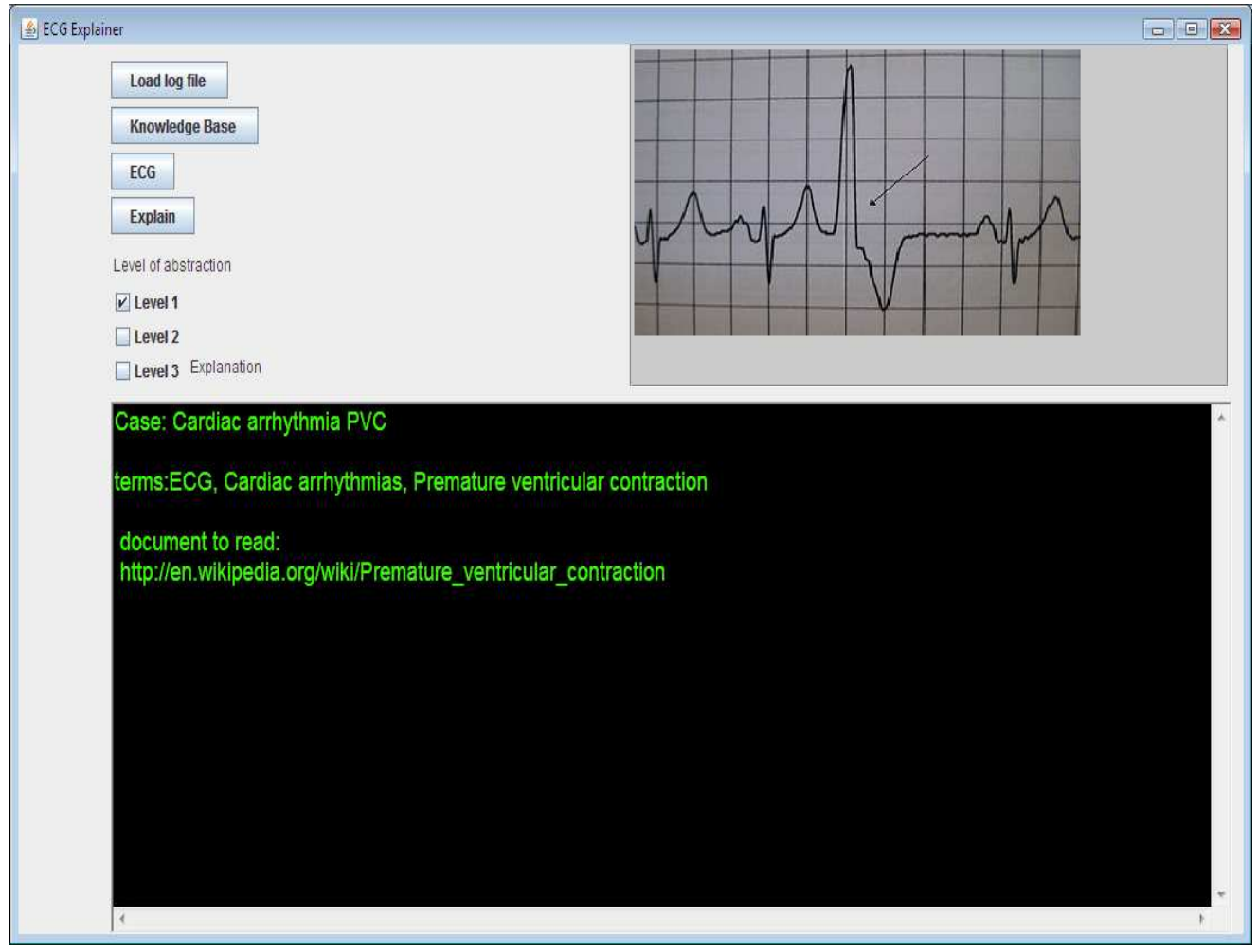

Fig.7. Example of explanation in level 1 of PVC arrhythmia.

\section{- Level 2}

In this level of abstraction, deep explanations are expected by medical doctors. After the analyze step and inferring step the agent visualizes the same explanation as in level 1, but the agent adds other kinds of explanations; for the justifications of the generated decision as in for example Fig 8, the agent visualizes the abnormal features. 
Medical Technologies Journal, Volume: 3, Issue: 2, April-June 2019, Pages: 376-394. Doi :

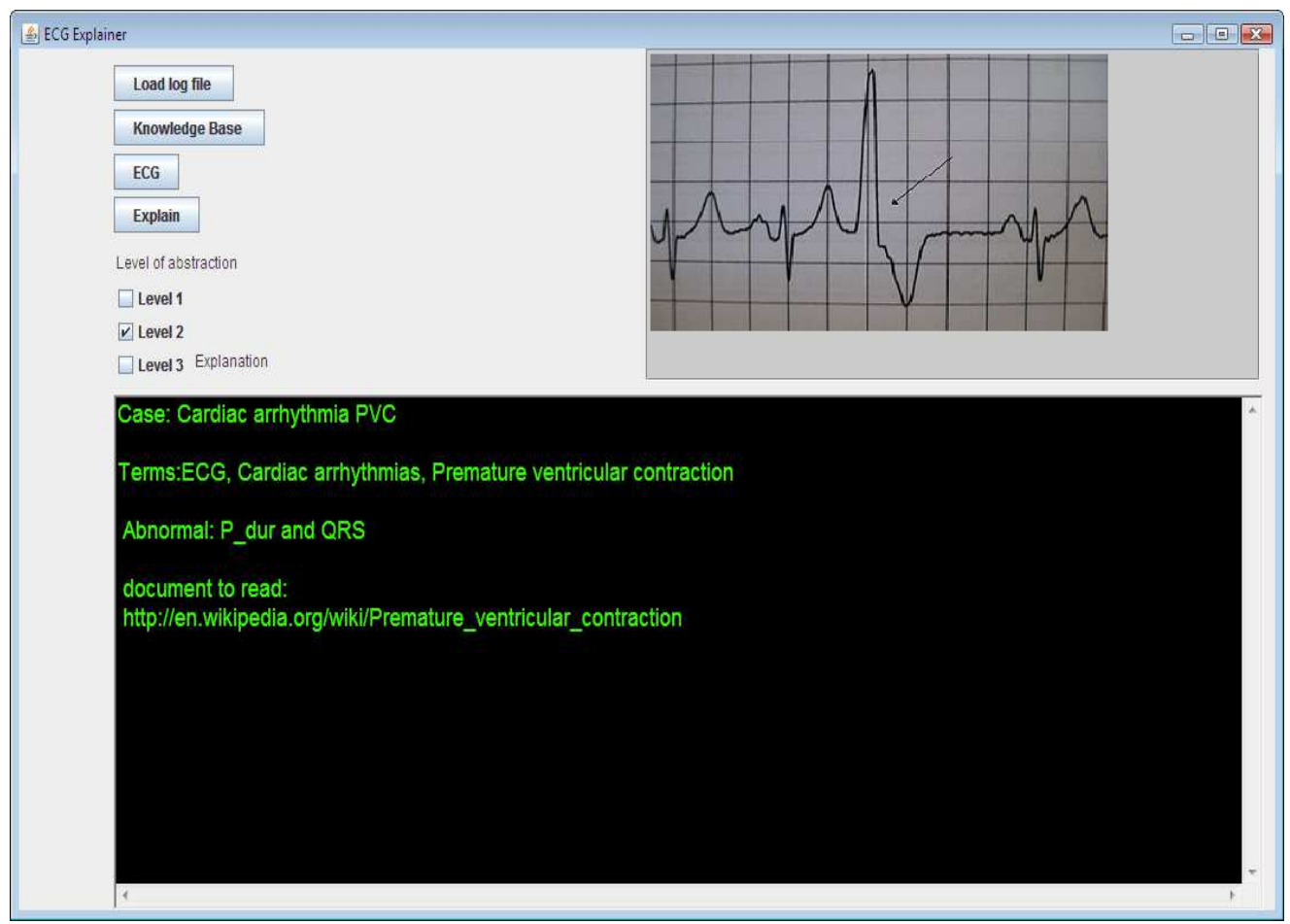

Fig.8. Example of explanation in level 2 of PVC arrhythmia.

\section{- Level 3}

In this level, all kinds of explanations are visualized; this level is designed for developers and experts. The agent presents the arrhythmia, the reasoning traces, the strategy of reasoning, the justifications, and the terminological explanation. This level of abstraction helps the developers to understand the abnormality inferred by the reasoning system for evaluation and maintenance. 


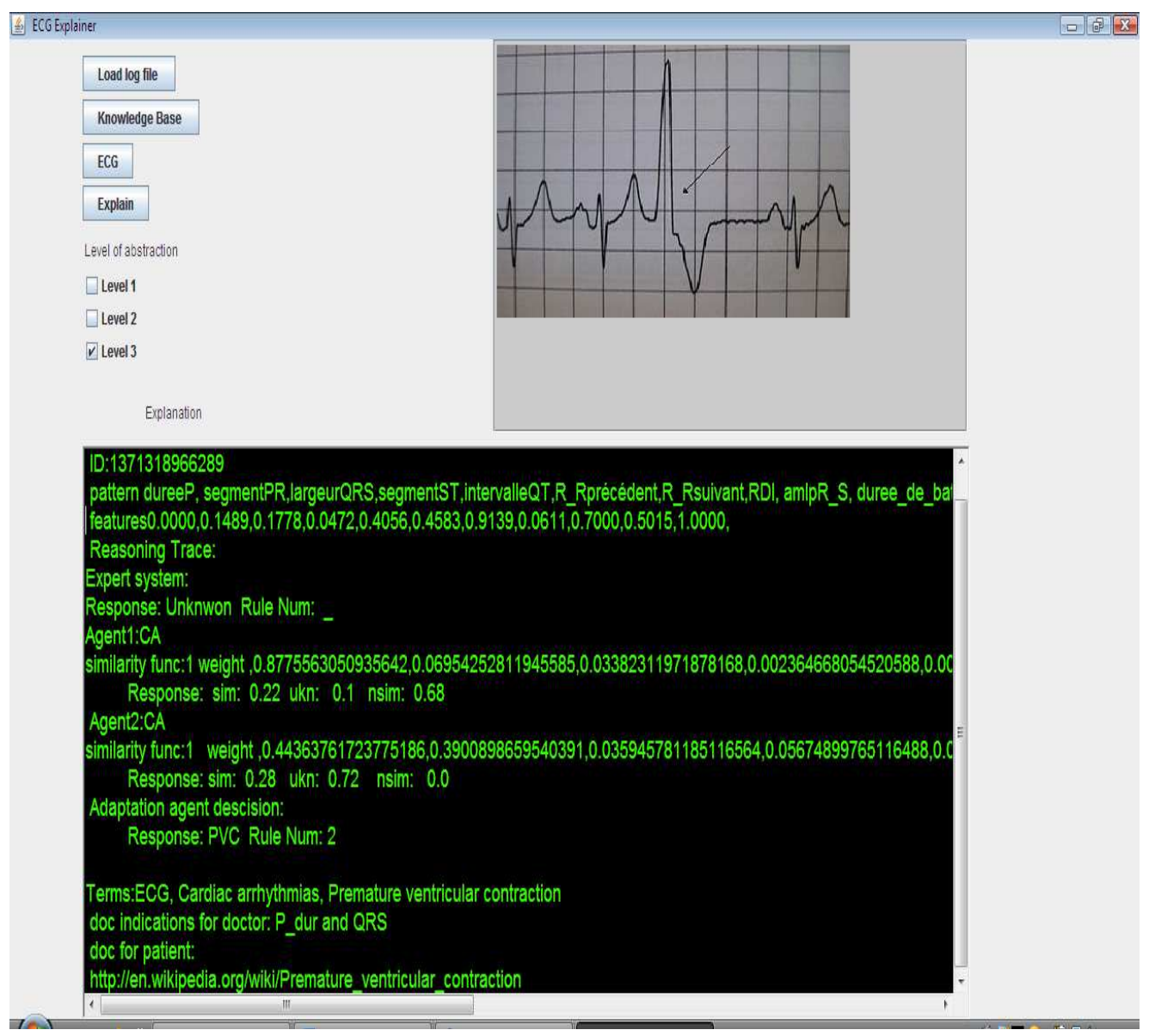

Fig.9. Example of explanation in level 3 of PVC arrhythmia.

As mentioned above, the accuracy of the decision support system depends on the extracted knowledge used and the applied similarity measures, for the mentioned reasons, the role of the explanation becomes crucial in the exploration cycle.

The physiological signal ECG could be visualized in all levels, but the characterization appears only in Level 3. This distinction is due to the complexity of the characterized information.

Figs 7-9 illustrate an example of an explanation in which the IK-DCBRC generates the PVC cardiac arrhythmia class for the introduced cardiac beat. In Fig 6 , the response associated with terminological explanations and document links is visualized when Level 1 is selected. On the second level of explanation, the agent adds the justification of the arrhythmia, in Fig 8, the cardiac beat characteristics imply that "the P and the QRS durations are abnormal".

In Fig 9, all kinds of explanation exist; the user can detect some conflicts. In the visualized explanations, the decision inferred from the rule-based system is unknown. The adaptation agent response is, in this example, the PVC class by using rule number 2 of the adaptation knowledge base. The rates of membership in similar, unknown and not similar fuzzy sets, of each similarity agent, are presented. Each similarity function used by the agents is visualized, function number 1, which refers to the sigmoid, with the appropriate weights vector, represents the importance degree of the attributes as a result of a learning process. 
In this example, a conflict can be detected, where the rate of membership in a similar set is so close: $22 \%$ for the normal class and $28 \%$ for the PVC class. However, the rate of membership in the not similar set is $68 \%$ for the normal class and $0.0 \%$ for the PVC class. This last observation decreases the uncertainty of the obtained conclusion and gives more trust.

In the literature, forty cardiac arrhythmias are defined, which justifies the use of an external knowledge base specializing in explanation for greater flexibility of the system

\section{Evaluation of the explanation agent}

In this step, we have used some well diagnosed cardiac beats, which are classified by BIH doctors and IK-DCBRC. We have used the same queries as Experiment 3 described in Table1. According to the trace of reasoning visualized by the explanation agent and the observation of real domain experts, we have defined three levels of risks (low, medium, and high). Table 4 summarizes the results of the explanation and the concluded results.

Table 4. The results of explanation by measuring risks after the explanation process with the same parameters of experiment 3 (see table 1).

\begin{tabular}{|l|l|l|l|l|l|}
\hline Class & Number of instances & Low risk & Medium risk & High risk & Total \\
\hline Normal & 100 & 52 & 10 & 38 & $50 \%$ \\
\hline PVC & 100 & 100 & 0 & 0 & $50 \% \%$ \\
\hline Total & 200 & $76 \%$ & $5 \%$ & $19 \%$ & $100 \%$ \\
\hline
\end{tabular}

Although IK-DCBRC well recognizes all queries, just $76 \%$ of them are classified with low risk and $5 \%$ with medium risk. The high risk in classification, which is $19 \%$, represents critical cases in which the similarity or dissimilarity is relatively equal, or the unknown is high.

\section{Conclusion}

The pragmatism and transparency of intelligent systems in medical domains and diagnosis are prominent and active research areas. The main achievement in this research work is focused on applying explanation-aware component for explaining the cardiac arrhythmia diagnosis supported by IK-DCBRC. The awareness of the explainer was introduced by ensuring an appropriate explanation for categorized users associated with a multi-level abstraction of explanation and visualization. The evaluation improves the importance of the proposed component for developing a strong relationship with users by recognizing the risk level of each decision.

The awareness parameter used in the selection of the level of abstraction is capitalized only by the satisfaction of users in his interaction with the explainer. Going forward, we believe that other external informational elements [40] about users influence the appropriation and transparency of the explanation as users' social identity. 


\section{Conflict of interest statement}

We certify that there is no conflict of interest with any financial organization in the subject matter or materials discussed in this manuscript.

\section{Authors' biographies}

Dr Abdeldjalil Khelassi: is an Associate Professor at Tlemcen University, Algeria. He obtained his Doctor in Science (2013), Magister (2008) and Engineer (2004) in Computer Sciences from the Department of Computer Science at Tlemcen University. His research interest includes cognitive systems, knowledge-based systems, case-based reasoning, distributed reasoning, fuzzy sets theory and health science. He is the editor manager of Medical Technologies Journal and the co-editor in chief of Electronic Physician Journal.

Dr Vania V. Estrela: B.Sc. degree from Federal University of Rio de Janeiro (UFRJ) in Electrical and Computer Engineering (ECE); M.Sc. in ECE from the Technological Institute of Aeronautics (ITA) and Northwestern University, USA; and Ph.D. in ECE from the Illinois Institute of Technology (IIT), Chicago, IL, USA. Taught at: DeVry University; State University of Northern Rio de Janeiro (UENF), Rio de Janeiro (RJ), Brazil; and for the West Zone State University (UEZO), RJ. Research interests include signal/image/video processing, inverse problems, computational \& mathematical modeling, stochastic models, multimedia, electronic instrumentation, machine learning and remote sensing. Reviewer for: IMAVIS (Elsevier); Pattern Recognition (Elsevier); COMPELECENG (Elsevier); Measurement (Elsevier); IET Image Processing; EURASIP Journal on Advances in Signal Processing (JASP) (Springer); IEEE Journal of Biomedical and Health Informatics (JBHI); Int'1 J. of Electrical and Comp. Engineering (IJECE); Int'l Journal of Ambient Computing and Intelligence (IJACI); Journal of Microwaves, Optoelectronics and Electromagnetic Applications (JMOE); and SET Int'l J. Broadcast Eng. (SET-IJBE). Engaged in topics such as technology transfer, STEM education, environmental issues and digital inclusion. Member of IEEE, and ACM. Editor of IJACI, EURASIP JASP, and SET-IJBE.

Dr. D. Jude Hemanth: received his B.E degree in ECE from Bharathiar University in 2002, M.E degree in communication systems from Anna University in 2006 and $\mathrm{Ph} . \mathrm{D}$. from Karunya University in 2013. His research areas include Computational Intelligence and Image processing. He has authored more than 100 research papers in reputed SCIE indexed/Scopus indexed International Journals and International Conferences with leading publishers such as Elsevier, Springer, IEEE, etc. His Cumulative Impact Factor is more than 50. He has authored 1 book with (VDMVerlag, Germany) and 11 edited books with reputed publishers such as Elsevier, Springer, IET and IOS Press. 


\section{References}

1. Hannah Ritchie and Max Roser (2018) - "Causes of Death". Published online at OurWorldInData.org. Retrieved from: 'https://ourworldindata.org/causes-of-death' [Online Resource]

2. Khelassi, A., Yelles-chaouche, S. N., \& Benais, F. (2017). Multi-arrhythmias detection with an XML rule-based system from 12-Lead Electrocardiogram. Electronic physician, 9(5), 4357. https://doi.org/10.19082/4357 PMid:28713507 PMCid:PMC5498700

3. Abdeldjalil KHELASSI, Mohamed Amin Chick. Fuzzy knowledge-intensive case based classification for the detection of abnormal cardiac beats. Electronic Physician, 2012;4(3):565571.

4. Smisek, R., Hejc, J., Ronzhina, M., Nemcova, A., Marsanova, L., Kolarova, J., ... \& Vitek, M. (2018). Multi-stage SVM approach for cardiac arrhythmias detection in short single-lead ECG recorded by a wearable device. Physiological Measurement. https://doi.org/10.1088/13616579/aad9e7 PMid:30102239

5. Xiong, Z., Nash, M. P., Cheng, E., Fedorov, V. V., Stiles, M. K., \& Zhao, J. (2018). ECG signal classification for the detection of cardiac arrhythmias using a convolutional recurrent neural network. Physiological Measurement. https://doi.org/10.1088/1361-6579/aad9ed PMid:30102248

6. Klir, G.J. and B. Yuan. (1995). Fuzzy Sets and Fuzzy Logic. Theory. and Applications, Ed. Prentice-Hall.

7. Aamodt, Agnar, and Enric Plaza. "Case-based reasoning: Foundational issues, methodological variations, and system approaches." AI communications 7.1 (1994): 39-59.

8. Hadjiski, M., Grancharova, A., \& Boshnakov, K. (2018). Intelligent Two-Level Optimization and Model Predictive Control of Degrading Plants. In Learning Systems: From Theory to Practice (pp. 117-134). Springer, Cham. https://doi.org/10.1007/978-3-319-75181-8_6

9. Frode Sørmo, Jörg Cassens, and Agnar Aamodt "Explanation in Case-Based Reasoning Perspectives and Goals" Artificial Intelligence Review, 24(2):109-143, October 2005. https://doi.org/10.1007/s10462-005-4607-7

10. John R. Hampton, 2013, The ECG Made Easy, Churchill Livingstone8 edition (August 27, 2013). ISBN-10: 0702046418.

11. Acharya, U. R., Hagiwara, Y., Koh, J. E. W., Oh, S. L., Tan, J. H., Adam, M., \& San Tan, R. (2018). Entropies for automated detection of coronary artery disease using ECG signals: A review. Biocybernetics and Biomedical Engineering. https://doi.org/10.1016/j.bbe.2018.03.001 12. Pan J, Tompkins WJ, A real-time QRS detection algorithm, IEEE Trans Biomed Eng 32:230-236, 1985. https://doi.org/10.1109/TBME.1985.325532 PMid:3997178

13. Berwal, D., Kumar, A., \& Kumar, Y. (2018). Design of high performance QRS complex detector for wearable healthcare devices using biorthogonal spline wavelet transform. ISA transactions.https://doi.org/10.1016/j.isatra.2018.08.002 PMid:30104037

14. Sotelo, S., Arenas, W., \& Altuve, M. (2018, April). QRS complex detection based on continuous density hidden Markov models using univariate observations. In Journal of Physics: Conference Series (Vol. 1002, No. 1, p. 012009). IOP Publishing. https://doi.org/10.1088/17426596/1002/1/012009

15. Ouali, M. A., Ghanai, M., \& Chafaa, K. (2018). A new type-2 fuzzy modelling and identification for electrophysiological signals: a comparison between PSO, BBO, FA and GA approaches. International Journal of Modelling, Identification and Control, 29(2), 163-184. https://doi.org/10.1504/IJMIC.2018.10011613

16. Rai, H. M., \& Chatterjee, K. (2018). A Novel Adaptive Feature Extraction for Detection of Cardiac Arrhythmias Using Hybrid Technique MRDWT \& MPNN Classifier from ECG Big Data. Big data research. https://doi.org/10.1016/j.bdr.2018.02.003

17. Zhaohao Sun, Jun Han, and Dong Dong (2008). Five Perspectives on Case Based Reasoning. In: D.-S. Huang et al. (Eds.): ICIC 2008, LNAI 5227, pp. 410-419, 2008.

18. Plaza, Enric; McGinty, Lorraine (2006). Distributed case-based reasoning. The Knowledge engineering review, Vol. 20, N. 3, pp. 261-265. https://doi.org/10.1017/S0269888906000683 
19. González-Briones, A., Prieto, J., De La Prieta, F., Herrera-Viedma, E., \& Corchado, J. M. (2018). Energy optimization using a case-based reasoning strategy. Sensors, 18(3), 865. https://doi.org/10.3390/s18030865 PMid:29543729 PMCid:PMC5877327

20. Miller, K., \& Mansingh, G. (2017). OptiPres: a distributed mobile agent decision support system for optimal patient drug prescription. Information Systems Frontiers, 19(1), 129-148. https://doi.org/10.1007/s10796-015-9595-9

21. Cho, B., Kim, K. J., \& Chung, J. W. (2017). CBR-based network performance management with multi-agent approach. Cluster Computing, 20(1), 757-767. https://doi.org/10.1007/s10586-017-0762-2

22. Aissani, N., Guetarni, I. H. M., \& Zebirate, S. (2017). Dynamic control for safety system multi-agent system with case-based reasoning. International Journal of Reliability and Safety, 11(3-4), 238-255. https://doi.org/10.1504/IJRS.2017.089708

23. A. Khelassi (2012). "Distributed Case Based Reasoning for Cardiac Arrhythmias: Health sciences application" LAMBERT ACADEMIC PUBLISHING -LAP-.

24. A.khelassi: "Data mining application with case based reasoning classifier for breast cancer decision support". MASAUM Inter Conf on Info Tech 2012 MI-CIT'12; 07/2012

25. A.Khelassi, "Fuzzy Case Based Classification applied in the classification of breast cancer pattern" Doctorial STIC11, TEBASSA, Algeria, 2011.

26. Khelassi, MA Chikh "Fuzzy knowledge-intensive case based classification applied in the automatic cardiac arrhythmias diagnosis" Colloque sur L'Optimisation et les Systèmes d'Information COSI 2011 P460, 24 -27 Avril, Guelma, Algérie

27. A.KHELASSI "Fuzzy Similarity Based classification Versus Intensive Knowledge Case Based classification" Colloque International sur les Nouvelles Techniques Immuno-Cognitive dans les Réseaux Informatiques NTICRI '09, Oran, Algeria

28. A.KHELASSI, M A CHIKH «Distributed classification using Intensive Knowledge Case Based Reasoning" Conférence Internationale des Technologies de l'Information et de la Communication CITIC'2009. Setif Algeria.

29. Abdeldjalil Khelassi, Mohamed Amin Chikh: Cognitive amalgam with a distributed fuzzy case-based reasoning system for an accurate cardiac arrhythmias diagnosis. Int. J. Information $\begin{array}{lllll}\text { and Communication } & \text { Technology } & \text { IJICT. } & \text { 07/2015; } & \text { 7(4/5):348-365. }\end{array}$ https://doi.org/10.1504/IJICT.2015.070297

30. A.KHELASSI, M A CHIKH " Distributed case based reasoning for cardiac arrhythmias diagnosis" MCSEAI 2008 Maghrebian Conference of Software Engineering and Artificial Intelligence, 28- 30 April 2008, Oran, Algérie,

31. Khelassi, A. (2015, January). The impact of uncertainty measures in the cardiac arrhythmia detection supported by IK-DCBRC. In Proceedings of the International Conference on Information and Knowledge Engineering (IKE) (p. 144). The Steering Committee of The World Congress in Computer Science, Computer Engineering and Applied Computing (WorldComp). 32. KHELASSI, Abdeldjalil. Explanation-aware computing of the prognosis for breast cancer supported by IK-DCBRC: Technical innovation. Electronic Physician, 2014, vol. 6, no 4, p. 947.

33. Agnar Aamodt. A Knowledge-Intensive, Integrated Approach to Problem Solving and Sustained Learning. PhD thesis, Norwegian Institute of Technology, Department of Computer Science, Trondheim, May 1991.

34. Thomas R. Roth-Berghofer and Jörg Cassens "Mapping Goals and Kinds of Explanations to the Knowledge Containers of Case-Based Reasoning Systems", ICCBR 2005, volume 3630 of LNAI, pages 451-464, Chicago, 2005. Springer. https://doi.org/10.1007/11536406_35

35. William J. Clancey. The epistemology of a rule-based expert system: A framework for explanation. Artificial Intelligence, 20(3):215-251, 1983. https://doi.org/10.1016/00043702(83)90008-5

36. William R. Swartout. What kind of expert should a system explain: A system for creating and explaining expert consulting programs. Artificial Intelligence, 21:285-325, 1983. https://doi.org/10.1016/S0004-3702(83)80014-9

37. William R. Swartout and Stephen W. Smoliar. On making expert systems more like experts. Expert Systems, 4(3):196-207, 1987. https://doi.org/10.1111/j.1468-0394.1987.tb00143.x 
Medical Technologies Journal, Volume: 3, Issue: 2, April-June 2019, Pages: 376-394. Doi :

https://doi.org/10.26415/2572-004X-vol3iss2p376-394

38. Goh, Y. M., \& Guo, B. H. (2018). FPSWizard: A web-based CBR-RBR system for supporting the design of active fall protection systems. Automation in Construction, 85, 40-50. https://doi.org/10.1016/j.autcon.2017.09.020

39. LIND, Linnéa Karlsson, VON EULER, Mia, KORKMAZ, Seher, et al. Sex differences in drugs: the development of a comprehensive knowledge base to improve gender awareness prescribing. Biology of sex differences, 2017, vol. 8, no 1, p. 32.https://doi.org/10.1186/s13293017-0155-5 PMid:29065918 PMCid:PMC5655861

40. Khelassi, A. (2016, January). An augmented pragmatics by explanation-aware and recommendation-aware in the context of decision support. In Proceedings of the International Conference on Information and Knowledge Engineering (IKE) (p. 79). The Steering Committee of The World Congress in Computer Science, Computer Engineering and Applied Computing (WorldComp). 